\title{
Impact of volume transition on the net charge of poly- $N$-isopropyl acrylamide microgels
}

\author{
M. Braibanti, ${ }^{1}$ C. Haro-Pérez, ${ }^{2, *}$ M. Quesada-Pérez, ${ }^{3}$ L. F. Rojas-Ochoa, ${ }^{4}$ and V. Trappe ${ }^{1, \dagger}$ \\ ${ }^{1}$ Department of Physics, University of Fribourg, CH-1700 Fribourg, Switzerland \\ ${ }^{2}$ Departamento de Ciencias Básicas, Universidad Autónoma Metropolitana-Azcapotzalco, Avenida San Pablo 180, \\ 02200 Ciudad de México, Mexico \\ ${ }^{3}$ Departamento de Física, Universidad de Jaén, Escuela Politécnica Superior de Linares, 23700 Linares, Spain \\ ${ }^{4}$ Departamento de Física, CINVESTAV-IPN, Avenida Instituto Politécnico Nacional 2508, 07360 Ciudad de México, Mexico
}

(Received 14 March 2016; published 6 September 2016)

\begin{abstract}
We explore the electrostatic properties of poly- $N$-isopropyl acrylamide microgels in dilute, quasi-de-ionized dispersions and show that the apparent net charge of these thermosensitive microgels is an increasing function of their size, the size being conveniently varied by temperature. Our experimental results obtained in a combination of light scattering, conductivity, and mobility experiments are consistent with those obtained in Poisson-Boltzmann cell model calculations, effectively indicating that upon shrinking the number of counterions entrapped within the microgels increases. Remarkably, this behavior shows that the electrostatic energy per particle remains constant upon swelling or deswelling the microgel, resulting in a square root dependence of the net charge on the particle radius.
\end{abstract}

DOI: 10.1103/PhysRevE.94.032601

\section{INTRODUCTION}

A key feature that distinguishes microgels from plain colloidal particles is their permeability. These soft colloids can absorb a large amount of water, where the extent of swelling often depends on environmental conditions, such as temperature and/or $p \mathrm{H}$; this stimulus responsiveness makes microgels excellent candidates for biotechnological applications, such as biomaterials and drug delivery [1-6]. Evidently, the uptake of small charged drugs by microgels is strongly conditioned by electrostatic forces. Moreover, electrostatic interactions play a crucial role in the colloidal stabilization and phase behavior of charged microgel systems $[7,8]$. To fully control these processes understanding the electrostatics of microgels is thus essential.

Such electrostatics will obviously depend on the charge coming from the ionized groups of the microgel, to which we will refer as bare charge $Z$. However, small ions can flow into the microgel, such that electrostatic interactions between nonoverlapping microgels are expected to depend on the net charge $Z_{\text {net }}$, which is not only determined by the number of ionized groups but also by the counterions located within the microgel, the net charge being defined as the bare charge minus the number of counterions within the microgel. For the description of the particle-particle interactions we need to further consider that the particles with a given net charge are dressed with the counterions that are located in the aqueous medium outside the microgels. This defines the effective charge $Z_{\text {eff }}$, determining the effective interaction potential that governs the phase behavior of the microgel system.

Theoretically only a few attempts have been made to describe the net and/or effective charge of microgels by considering the penetration of ions into the gel network to properly account for the electrical double layer of microgels [8-16]. Among these attempts, Denton derived an expression for the interaction energy between charged microgels

\footnotetext{
*cehp@azc.uam.mx

${ }^{\dagger}$ veronique.trappe@unifr.ch
}

in the linear screening regime, whose functional form for nonoverlapping particles is similar to the classical Yukawa electrostatic interaction potential of plain colloids [8]. A result of this theory is that the net charge increases when the particle radius decreases due to a reduction of the number of counterions within the microgel $[8,12]$. In agreement with this prediction, numerical simulations of nanogels with strong internal excluded volume interactions show that counterions are expelled from inside of the particles as the available volume for counterions is reduced [17]. In the case of thermosensitive microgels exhibiting a volume phase transition upon increasing temperature, we would thus expect that the effective charge should increase upon heating. Results obtained by modeling the temperature dependence of electrophoretic mobility of thermosensitive microgels with Ohshima theory seemed to agree with these predictions [18]. However, recent experiments exploring the structural properties of such microgel systems [19] and primitive model calculations [16] showed that the effective microgel charge instead decreases with decreasing particle size, i.e., increasing temperature.

In this contribution we further investigate the effect of the volume phase transition on the electrostatic interactions of poly- $N$-isopropyl acrylamide (PNiPAM) microgels in dilute, quasi-de-ionized dispersions and show that the effective charge decreases upon microgel collapse due to a decrease of the net charge; i.e., the number of counterions trapped within the microgel increases as the microgel size decreases with increasing temperature. Poisson-Boltzmann cell model calculations reproduce our data remarkably well and the combination of our experimental and theoretical findings reveals that for permeable spheres with a given bare charge the electrostatic energy per particle is preserved upon varying the particle size.

\section{SAMPLE CHARACTERISTICS AND EXPERIMENTAL TECHNIQUES}

\section{A. Sample characteristics}

PNiPAM particles are synthesized in dispersion polymerization as described by Senff and Richtering [20]. In a round- 
bottom flask, equipped with a stirrer, a reflux condenser, and a gas inlet, $7.9 \mathrm{~g}$ of monomer $N$-isopropyl acrylamide (NiPAM), $0.17 \mathrm{~g}$ of the cross-linker $N, N$-methylenebisacrylamide (BIS), and $0.15 \mathrm{~g}$ of the stabilizer sodium dodecylsulfate (SDS) are dissolved in $450 \mathrm{ml}$ pure water under stirring. Separately, a solution of $0.6 \mathrm{~g}$ of the initiator potassium peroxodisulfate (KPS) in $50 \mathrm{ml}$ of pure water is prepared. After bubbling both solutions with nitrogen for $30 \mathrm{~min}$, the initiator is added to the monomer solution that has been previously heated to $70^{\circ} \mathrm{C}$. After $5 \mathrm{~h}$, the dispersion is cooled down and purified by filtration through glass wool and extensive dialysis against pure water.

Because of the use of an ionic initiator our PNiPAM microgels are negatively charged, such that their interactions are sensitive to ionic strength. In the course of our investigations we found that the standard procedure of purification by dialysis is not efficient in removing a compound that significantly alters the phase behavior of the microgels at quasi-de-ionized conditions. This compound can be removed by repeated centrifugation followed by an exchange of the supernatant with pure water, which suggests that the standard synthesis described above yields some larger polymeric entities that are less dense than the microgels, such that they can be removed by repeated centrifugation steps but not by dialysis. In this work we focus on the results obtained after five centrifugation steps. The temperature dependent characteristics of the effective charge described in the following are, however, independent of the degree of purity of the sample; i.e., they are independent of the number of centrifugation steps used and can be observed even for systems that have not been purified by centrifugation.

To maximize the electrostatic interactions among the microgels we work at quasi-de-ionized conditions. To achieve this, our microgel samples are sealed in quartz cuvettes that contain a small amount of ionic exchange resin (Amberlite IRN 150, Fluka), which exchanges both cation and anion with, respectively, $\mathrm{H}^{+}$and $\mathrm{OH}^{-}$. Samples with different concentrations are produced by dilution of a stock solution. To determine the particle number concentration $n$ of each sample we exploit the fact that for charged colloids at low ionic strength the main peak position of the static structure factor $q_{\text {max }}$ is strongly determined by $n[21,22]$. In the case of samples with structure factors typical of fluid systems, we use $n$ and the effective charge of the particle $Z_{\text {eff }}$ as fitting parameters in order to reproduce the experimental $q_{\max }$ by solving the Ornstein-Zernike equation using the hypernetted chain (HNC) closure relation, assuming a Yukawa-like potential [23] of the form $u(r)=\frac{Z_{\text {eff }}^{2} e^{2}}{4 \pi \varepsilon_{0} \varepsilon_{r}}\left[\frac{\exp (\kappa R)}{1+\kappa R}\right]^{2} \frac{\exp (-\kappa R)}{r}$ for $r>2 \mathrm{R}$ and a hard sphere potential for $r<2 \mathrm{R}$, where $r$ is the center to center particle distance, $R$ the particle radius, $e$ the electron charge, $\kappa$ the effective inverse Debye screening length, $\varepsilon_{0}$ the vacuum dielectric constant, and $\varepsilon_{r}$ the medium relative dielectric constant. In the case of crystalline samples, we determine first the crystal lattice from the relative positions of the measured Bragg peaks and then estimate the particle concentration from the main peak position [22]. The relative values of the concentrations obtained by determining the number concentration of each sample correspond well with those expected from the dilution procedure. The temperature dependent volume fraction of a given sample is calculated by multiplying the volume of the particle with the number concentration.

The state of a given system, fluid or crystal, is determined after an equilibration time of at least 1 week at the desired temperature, where we initially shear-rejuvenate the system at temperatures exceeding the lower critical solution temperature (LCST) to obtain a fully randomized configuration of the particle position as starting condition. The formation of crystals is visually observed by the appearance of small iridescent regions within the sample, where additional experiments in static light scattering confirm the formation of crystals, as evidenced by the appearance of Bragg peaks.

\section{B. Experimental techniques}

Light scattering experiments are performed by using a 3D dynamic light scattering (3D-DLS) setup spectrometer (LS Instruments AG, Fribourg, Switzerland) [24-27]. The 3D scheme is based on the realization of two simultaneous scattering experiments performed at scattering vectors $\boldsymbol{q}$ with equal magnitude but different directions. To achieve this, a laser beam (HeNe laser, Uniphase $21 \mathrm{~mW}, \lambda=632.8 \mathrm{~nm}$ ) is split into two beams that are off shifted in the vertical. Both beams are then deviated by a lens to impinge the sample from two different directions crossing each other in the scattering volume. A second lens on the arm of the goniometer redirects the scattered light onto single mode fibers connected to avalanche photodiode detectors (PerkinElmer) and a Flex correlator [28] is used to calculate the intensity cross-correlation function. Because of the $3 \mathrm{D}$ configuration only the single scattered light is correlated. The decay of the cross-correlation function thus reflects the structural relaxation at the chosen $q$-vector, even if the system is somewhat multiply scattering.

The microgel mobility $\mu_{M}$ is obtained by using a Brookhaven Zeta PALS device with the BI-ZEL Brookhaven cell for aqueous and polar liquids. The PALS (phase analysis light scattering) technique uses the phase shift caused by moving particles to measure their velocity. This phase shift is estimated by comparing the phase of the light scattered by the moving particles with the phase of a reference beam. Our mobility experiments are performed as a function of both temperature and salt concentration, using a microgel sample with a number concentration of $n=2 \mu \mathrm{m}^{-3}$. To estimate the mobility of the microgels at quasi-de-ionized conditions we extrapolate the data obtained at different ionic strengths to $10^{-6} M$, which has been determined previously to be the residual ionic strength of de-ionized water [29,30].

The temperature dependence of the conductivity of the microgel sample $\sigma_{S}$ with $n=2 \mu \mathrm{m}^{-3}$ and that of water $\sigma_{\mathrm{H}_{2} \mathrm{O}}$ is measured directly at quasi-de-ionized conditions using a YSI 3200 conductivity meter.

The combination of the mobility and conductivity results then allows us to determine an apparent net charge $Z_{\text {net,app }}$ by assuming that the conductivity is proportional to the total number of charges present in the system times the microgel mobility [31]:

$$
\begin{aligned}
\sigma_{S}-\sigma_{\mathrm{H}_{2} \mathrm{O}} & =n e Z_{\text {net, app }}\left(\mu_{M}+\mu_{\mathrm{H}^{+}}\right) \Rightarrow \\
Z_{\text {net, app }} & =\frac{1}{n e} \frac{\sigma_{S}-\sigma_{\mathrm{H}_{2} \mathrm{O}}}{\mu_{M}+\mu_{\mathrm{H}^{+}}},
\end{aligned}
$$


with $\mu_{\mathrm{H}^{+}}$the mobility of the protons, the temperature dependence of $\mu_{\mathrm{H}^{+}}$being known from literature [32]. Let us here note that in particular for highly charged particles $Z_{\text {net,app }}$ differs from the net charge. This is because strongly bound counterions are dragged along with the particle under the effect of an electric field. Consequently, the apparent net charge is smaller than the net charge of the microgels defined in the Introduction.

\section{EXPERIMENTAL RESULTS}

\section{A. Contributions of electrostatics to the swelling behavior of PNiPAM microgels}

PNiPAM is a neutral polymer and the electrostatic properties of PNiPAM microgels are generally due to the use of ionic initiators in the synthesis of these systems. It is thus reasonable to assume that the charges are always attached to dangling ends [33]. Moreover, because the PNiPAM synthesis is performed at a temperature that significantly exceeds the LCST, we can expect that the charges are mainly located at the outer edge of the microgels. Indeed, the globular state of PNiPAM should force a preferential orientation of the charges towards the aqueous medium during the synthesis, such that the microgels will have a charged corona [34].

Evidence for this is obtained by comparing the dimensions of the microgels at standard conditions to those obtained at quasi-de-ionized conditions, where we here denote a system that is not in contact with ion exchange resin as PNiPAM at standard conditions. As shown in Fig. 1, de-ionization leads to a sizable increase of the hydrodynamic radius $R_{h}$, in particular at low temperatures. With increasing temperature the microgel shrinks and finally collapses to a globular state at the LCST denoted as a vertical line. Beyond the LCST the hydrodynamic radii at both conditions are rather similar, but still distinguishable.

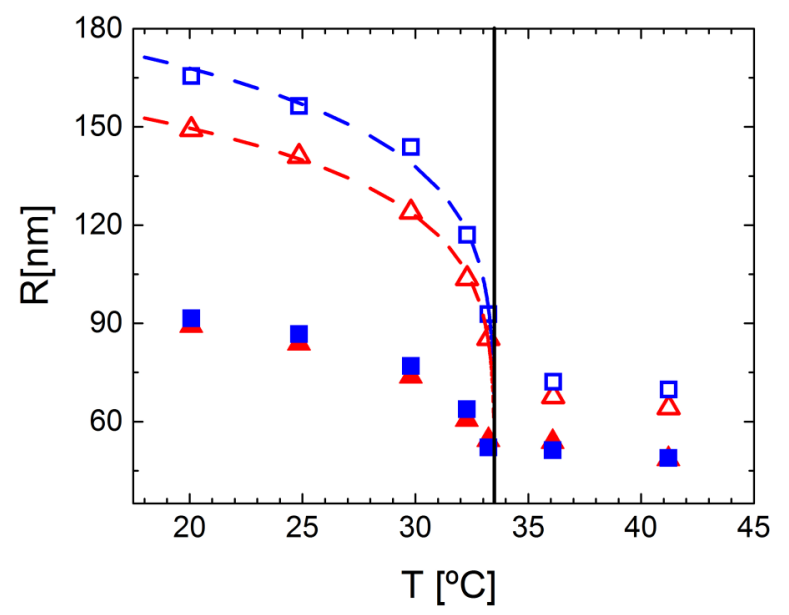

FIG. 1. Temperature dependence of the hydrodynamic radius (open symbols) and radius of gyration (solid symbols) of PNiPAM microgel at de-ionized (blue squares) and standard conditions (red triangles). The dotted lines through the data are fits to a critical-like function of form $R_{h}=A\left(T_{c}-T\right)^{b}$ with $T_{c}=33.5^{\circ} \mathrm{C}$ denoted by the vertical solid line.
As reported in different studies [35-37], the cross-linking density is not homogeneous within the PNiPAM microgels. Indeed, they can be considered as core-shell particles, where the core is highly cross-linked while the shell consists of less cross-linked material with more dangling ends. The highly cross-linked core gives rise to a higher optical contrast, such that the radius of gyration $R_{g}$ determined from static light scattering experiments can be related to the core dimensions, while $R_{h}$ is a measure of the overall dimensions of the microgels. That $R_{g}$ is not altered upon de-ionization suggests that the core contains only very little charges. The increase in $R_{h}$ upon de-ionization then in turn indicates that the increase of the overall microgel dimensions is mainly due to a swelling of the shell that contains most of the charges. This agrees with findings obtained in dielectric relaxation spectroscopy that led to the same conclusion, namely, that the charges are mainly located in the peripheral shell [34].

\section{B. Temperature dependence of the microgel effective charge}

To explore the impact of the volume phase transition of PNiPAM on the effective charge governing the electrostatic interactions between microgels we perform three different types of experiments. In a first set of experiments we determine the structure factor of a microgel system with a fixed number concentration of $n=0.77 \mu \mathrm{m}^{-3}$. At this concentration the system behaves as a correlated liquid, as evidenced by the liquid structure factor shown in Fig. 2(a). Upon varying the temperature from $20^{\circ} \mathrm{C}$ to $40^{\circ} \mathrm{C}$ the particle volume fraction $\phi$ varies from $1.45 \times 10^{-2}$ to $1.25 \times 10^{-3}$. Despite this rather significant variation in $\phi$ the peak position remains unchanged, which denotes that the electrostatic interactions are, at all temperatures investigated, sufficiently strong to lead to a configuration where the average distance among the microgels is maximized. However, the degree of correlation in the microgel suspension decreases with increasing temperature, as evidenced by the decrease in the peak height $S\left(q_{\max }\right)$ shown in Fig. 2(b). Such decrease in $S\left(q_{\max }\right)$ could be due to a reduction of the particle size and thus a reduction of the particle volume fraction and/or a reduction of the microgel effective charge. To address whether solely the temperature dependence of $\phi$ could lead to the decrease observed, we calculate the $\phi$ dependence of $S\left(q_{\max }\right)$ for a system with a fixed effective charge of $Z_{\text {eff }}=835$, a particle number concentration of $n=0.77 \mu \mathrm{m}^{-3}$, and a residual ionic strength of $10^{-6} M$ using the interaction potential and closure relation described in Sec. II A. The comparison of the calculation (dashed line) with the experimental findings (full symbols) reveals that $S\left(q_{\max }\right)$ decreases more significantly than expected, if the volume fraction would be the only parameter changing with temperature. To account for the observed decrease of $S\left(q_{\max }\right)$, we thus need to consider that $Z_{\text {eff }}$ is temperature dependent as well. To estimate $Z_{\text {eff }}$ we fit the experimental $S(q)$ leaving $Z_{\text {eff }}$ as a free parameter, taking the temperature dependence of volume fraction into account. The best fits reproducing $S(q)$ are shown as lines in Fig. 2(a). The resulting $Z_{\text {eff }}$ decreases with temperature, exhibiting a temperature dependence similar to that of $S\left(q_{\max }\right)$, as shown in Fig. 2(c).

The decrease of $Z_{\text {eff }}$ upon collapse of the microgels also impacts on their phase behavior. Indeed, at large enough 

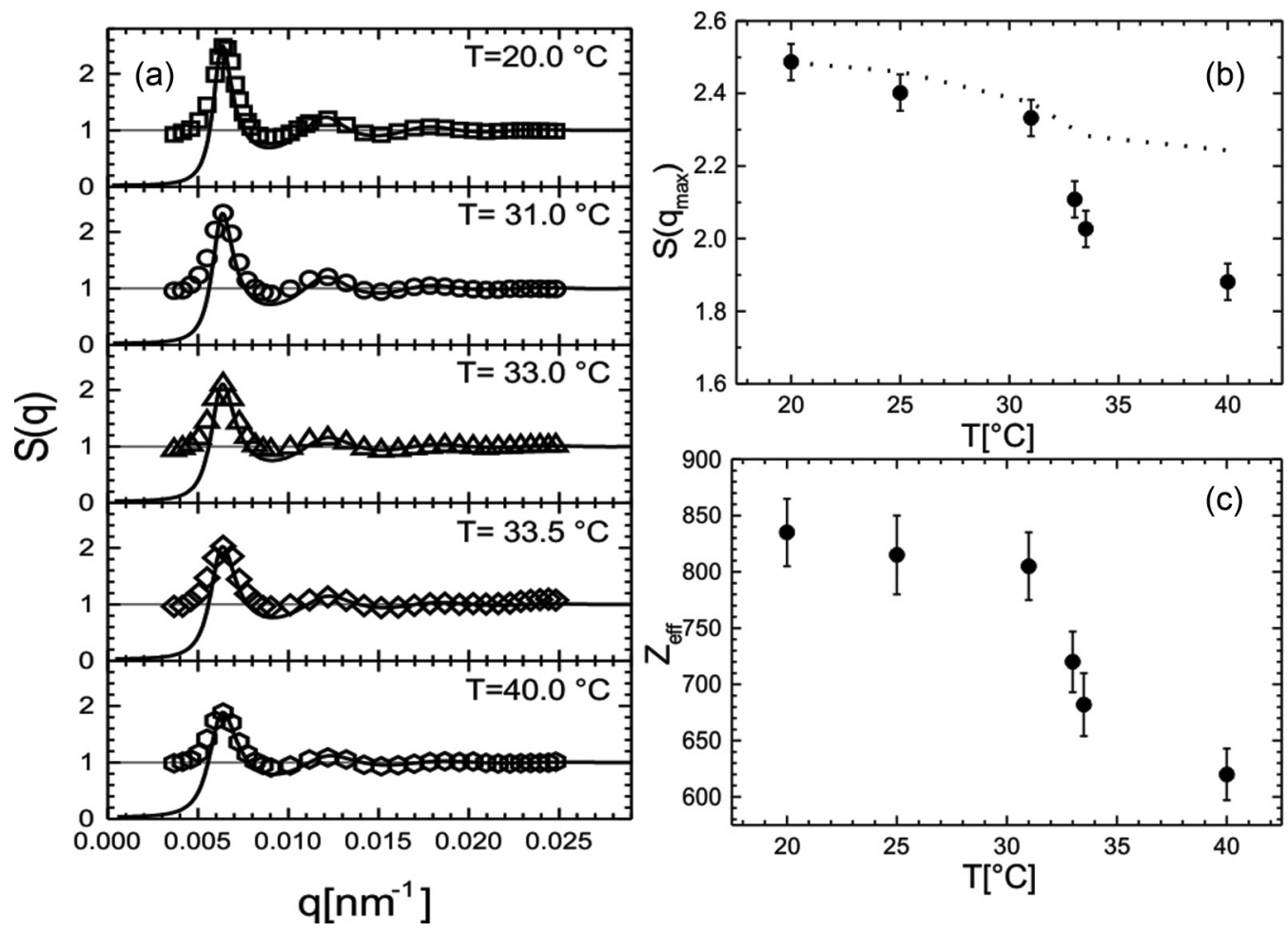

FIG. 2. (a) Static structure factor of a microgel system with $n=0.77 \mu \mathrm{m}^{-3}$ at temperatures as indicated in the graphs. Lines correspond to calculations obtained by solving the Ornstein-Zernike equation using the HNC-closure relation and assuming that the microgels interact by a Yukawa-like potential (see Sec. II A.) (b) Magnitude of the nearest neighbor peak as a function of temperature. Dashed line corresponds to the theoretical prediction assuming a constant particle charge $Z_{\text {eff }}$ of 835 taking only the temperature dependence of the particle size and thus the volume fraction into account. (c) Effective charge used in the calculations of the static structure factors plotted in (a) as a function of temperature.

concentrations we find that the microgels assemble into crystals. The higher the temperature, the smaller the volume of the microgel, the larger the number concentration needed to obtain crystalline states, as shown in Fig. 3(a), where we also denote a narrow range of concentration, where fluid and crystals coexist. We exploit the temperature dependence of the boundary between fluid and crystals indicated as a continuous line in Fig. 3(a) to evaluate $Z_{\text {eff }}$. We assume that $S\left(q_{\max }\right)$ reaches the value expected for freezing at the onset of crystallization according to the Hansen-Verlet criterion stating that a system can be considered as frozen when $S\left(q_{\max }\right)>2.85$ [38,39]. Thus, we impose $S\left(q_{\max }\right)=2.85$ at the boundary and calculate $Z_{\mathrm{eff}}$ following the procedure to calculate $S(q)$ explained above. In this calculation $Z_{\text {eff }}$ is the only free parameter, since the number concentration at the boundary and the temperature dependent size of the particles are known. The temperature dependence of the resulting $Z_{\text {eff }}$ shown in Fig 3(b) confirms the trend already observed in Fig. 2(c): $Z_{\text {eff }}$ is a decreasing function of temperature. However, let us note that the magnitude of $Z_{\text {eff }}$ is lower using the phase boundary criterion as a measure for $Z_{\text {eff }}$ than that obtained from the fit of the structure factor of the system at lower concentration.

The determination of the microgel charge so far is based on measures that depend on the particle interaction potential. A more direct measure of the particle charge can be obtained by combining the conductivity of the system with its electrophoretic mobility, as denoted in Eq. (1). As shown in Figs. 4(a) and 4(b) the magnitude of both conductivity and mobility are increasing with increasing temperature. At first sight this may be interpreted as that the particle net charge is an increasing function of temperature. However, the contributions of the particles alone to the conductivity of the system are decreasing with increasing temperature, as shown in the inset of Fig. 4(a). Indeed, calculating $Z_{\text {net, app }}$ according to Eq. (1) reveals a temperature dependence of $Z_{\text {net,app }}$ reminiscent of that of $Z_{\mathrm{eff}}$ : $Z_{\text {net,app }}$ is a decreasing function of temperature, as shown in Fig. 4(c). The magnitude of $Z_{\text {net,app }}$, however, significantly exceeds that of $Z_{\text {eff }}$.

At this point it is worth recalling that we found that the actual phase behavior of our microgel systems is rather sensitive to a nonidentified polymeric component that can only be removed by repeated exchange of the aqueous phase using a centrifugation and redispersion procedure. It is reasonable to assume that this "dirt" effect will not severely affect conductivity and mobility measurements and thus our determination of $Z_{\text {net,app }}$, while impacting our estimates of $Z_{\text {eff }}$ that are based on the phase behavior of the system. The significant discrepancy between $Z_{\text {eff }}$ and $Z_{\text {net,app }}$ is thus likely due to the fact that our systems are not entirely pure. Indeed, we find that further purification leads to changes in the phase behavior of the system consistent with a larger $Z_{\text {eff }}$, a $Z_{\text {eff }}$ that is now of the order of $Z_{\text {net,app }}$. Let us note that despite a somewhat problematic determination of the absolute magnitude of $Z_{\text {eff }}$, all our experiments denote that the temperature dependence of $Z_{\text {eff }}$ is independent of the degree of purity of the sample. 

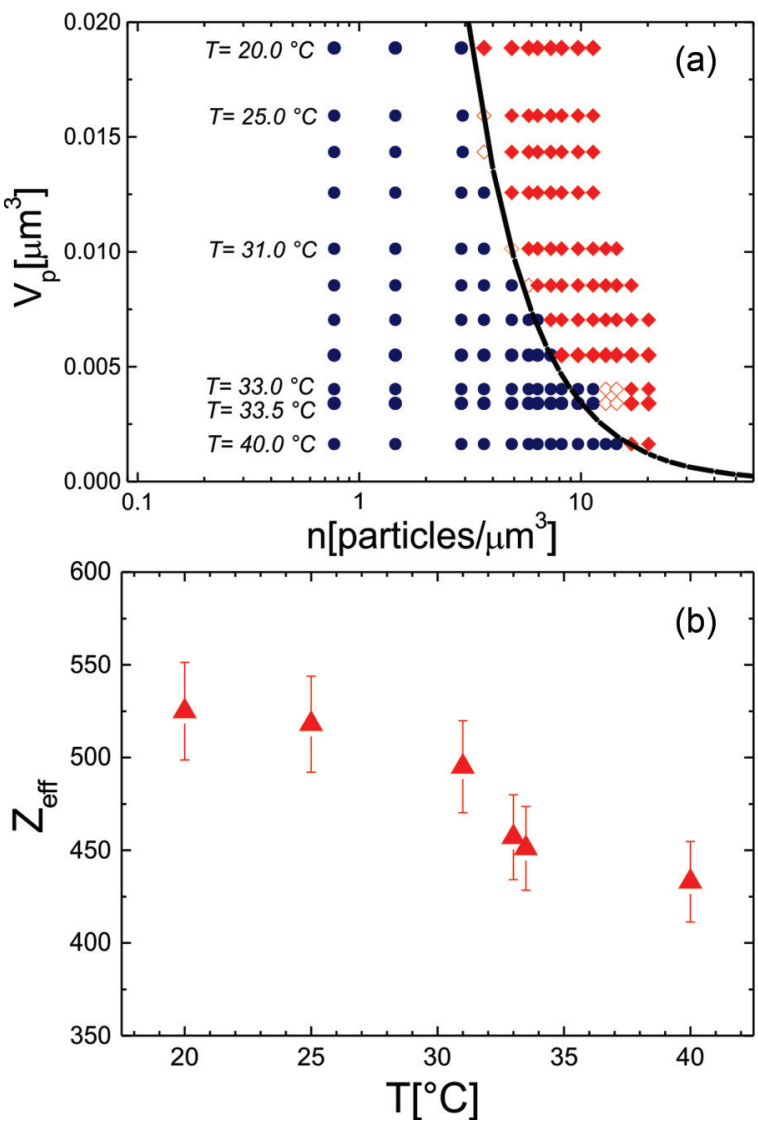

FIG. 3. (a) Dependence of the phase behavior of PNiPAM microgel systems at de-ionized conditions on number concentration $n$ and particle volume $V_{p}$, where $V_{p}=4 / 3 \pi R_{h}^{3}$ depends on temperature. Fluid samples are represented by solid circles and crystalline samples by solid diamonds. The coexistence of crystals and fluids is denoted as open diamonds. The solid line delimiting the phase space between liquid and crystalline states is a guide to the eye. (b) Temperature dependence of the effective charges obtained at the fluid crystal boundary, by assuming $S\left(q_{\max }\right)=2.85$.

In all systems investigated we find that there is a loss of effective charge associated to the volume phase transition of the PNiPAM-microgels upon increasing the temperature.

\section{THEORETICAL MODELING}

To account for the loss of charge related to the volume phase transition of our microgels we consider a model $[10,13]$ inspired by the Poisson-Boltzmann cell model (PB cell model), previously used by Alexander et al. for the description of charged impermeable colloids [40]. The basic assumptions of our model are (i) the counterion distribution is mainly governed by electrostatic interactions and thermal motion; (ii) the microgel is a permeable sphere whose charged groups are uniformly distributed and, therefore, its charge density is for a negatively charged microgel given by $-3 Z e / 4 \pi R^{3}$; (iii) the counterions can move through the microgel without undergoing significant excluded volume effects. Under these conditions, the electrostatic potential, $\psi(r)$, and the counterion concentration at a distance $r$ from the particle center, $n_{c}(r)$, can be obtained by solving the following Poisson-Boltzmann

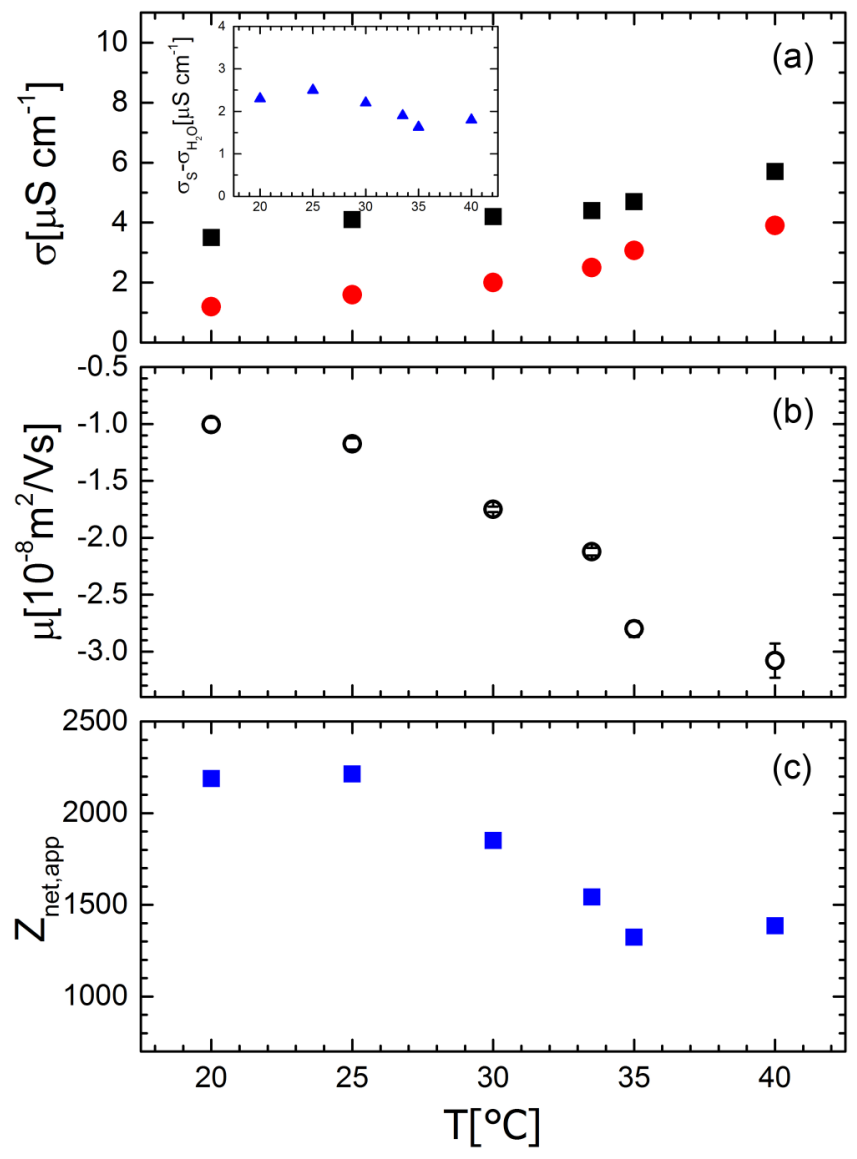

FIG. 4. (a) Temperature dependent conductivity of a microgel system with $n=2 \mu \mathrm{m}^{-3}$ (black squares $\sigma_{S}$ ) and pure water (red circles $\sigma_{\mathrm{H}_{2} \mathrm{O}}$ ) at quasi-de-ionized conditions. Inset: Difference $\sigma_{S}-$ $\sigma_{\mathrm{H}_{2} \mathrm{O}}$. (b) Temperature dependent electrophoretic mobility of the same microgel system, where we use an extrapolation procedure based on data obtained at different ionic strength to evaluate the mobility at quasi-de-ionized conditions.(c) Temperature dependence of the apparent net charge according to Eq. (1).

equation using

$$
\begin{aligned}
\Psi^{\prime \prime}(r)+\frac{2}{r} \Psi^{\prime}(r)= & -4 \pi l_{B} n_{c}\left(R_{\text {cell }}\right) \exp [-\Psi(r)] \quad(r>R), \\
\Psi^{\prime \prime}(r)+\frac{2}{r} \Psi^{\prime}(r)= & 4 \pi l_{B}\left\{3 Z / 4 \pi R^{3}-n_{c}\left(R_{\text {cell }}\right)\right. \\
& \times \exp [-\Psi(r)]\} \quad(r \leqslant R),
\end{aligned}
$$

where $\Psi(r)$ is the normalized electrostatic potential $\Psi(r)=$ $e \psi(r) / k_{B} T, l_{B}=e^{2} /\left(4 \varepsilon_{r} \varepsilon_{0} k_{B} T\right)$ is the Bjerrum length, $R_{\text {cell }}$ is the radius of the spherical PB cell, and $n_{c}\left(R_{\text {cell }}\right)$ is the counterion concentration just at the border of the cell. The radius of the cell can be estimated from the microgel particle concentration $n$ as $R_{\text {cell }}=(4 \pi n / 3)^{-1 / 3}$. Let us note that $n_{c}(r)=n_{c}\left(R_{\text {cell }}\right) \exp [-\Psi(r)]$ provided that $\Psi\left(R_{\text {cell }}\right)=0$.

Equation (2) is solved together with the boundary conditions $\Psi\left(R_{\text {cell }}\right)=0, \Psi^{\prime}\left(R_{\text {cell }}\right)=0$ (electroneutrality) and $\Psi^{\prime}(0)=0$ (spherical symmetry) using an iterative method similar to that described in [40] (for further details see [13]): 
(i) First, an initial guess for the electric field, $E(r)$, is proposed.

(ii) Then, the electrostatic potential is calculated by integrating the electric field:

$$
\psi(r)=\int_{R_{\text {cell }}}^{r} E(r) d r .
$$

(iii) The counterion concentration is then given by

$$
n_{c}(r)=\frac{Z}{\int_{0}^{R_{\text {cell }}} \exp [-e \psi(r)] 4 \pi r^{2} d r} \exp [-e \psi(r)] .
$$

(iv) From the ion profile, the charge density $\rho(r)$ is computed as

$$
\rho(r)=\left\{\begin{array}{l}
-\frac{e Z}{4 \pi R^{3} / 3}+e n_{c}(r) r \leqslant R \\
e n_{c}(r) r>R
\end{array}\right.
$$
by

(v) The net charge enclosed by a sphere of radius $r$ is given

$$
Q(r)=\int_{0}^{r} \rho(r) 4 \pi r^{2} d r .
$$

(vi) The electric field at a distance $r$ is recalculated applying Gauss's law:

$$
E(r)=\frac{Q(r)}{4 \pi \varepsilon_{0} \varepsilon_{r} r^{2}} .
$$

Having calculated the electric field, a new iteration begins. After solving the PB equation the net charge $Z_{\text {net }}$ of the microgel is then computed and expressed in absolute values of $Z_{\text {net }}=-Q(R) / e$.

To test this model for the description of the electrostatics of our microgels let us first focus on $Z_{\text {net,app }}$ obtained from combining mobility and conductivity measurements and compare these results with the corresponding $Z_{\text {net }}$ predictions of the PB cell model. Because the bare charge of the microgels is not known we proceed by reproducing the apparent net charge obtained from experiments at $40^{\circ} \mathrm{C}$ using the cell model, thereby fixing the bare charge to $Z=6000$. The other parameters needed for the calculation are known a priori. Using this bare charge the PB cell calculations reproduce the experimental $Z_{\text {net,app }}$ at all other temperatures reasonably well, as shown in Fig. 5(a). To test whether restricting the location of the charges within shells has a severe effect on our estimates, we calculate $Z_{\text {net }}$ for core-shell microgels using the shell thicknesses determined by Ledesma-Motolinía et al. for an equivalent microgel system [37]. Maintaining $Z=6000$ and assuming that the shell thickness over which the charge is distributed varies from $100 \mathrm{~nm}$ at $20^{\circ} \mathrm{C}$ to $30 \mathrm{~nm}$ at $40^{\circ} \mathrm{C}$, we find that $Z_{\text {net }}$ of the core-shell microgels would not deviate by more than $10 \%$ from the estimates obtained assuming a homogeneous distribution of charges. Such rather small dependence of $Z_{\text {net }}$ on the charge distribution agrees with results obtained in Monte Carlo coarse-grain simulations [13], which further validates the use of the cell model to predict the ionic profile outside the microgel and therefore its net charge even if the bare charges are unevenly distributed.

The PB cell model can also be used to describe the behavior of $Z_{\text {eff }}$. However, its application to highly charged systems requires the renormalization of the charge. Such renormalization
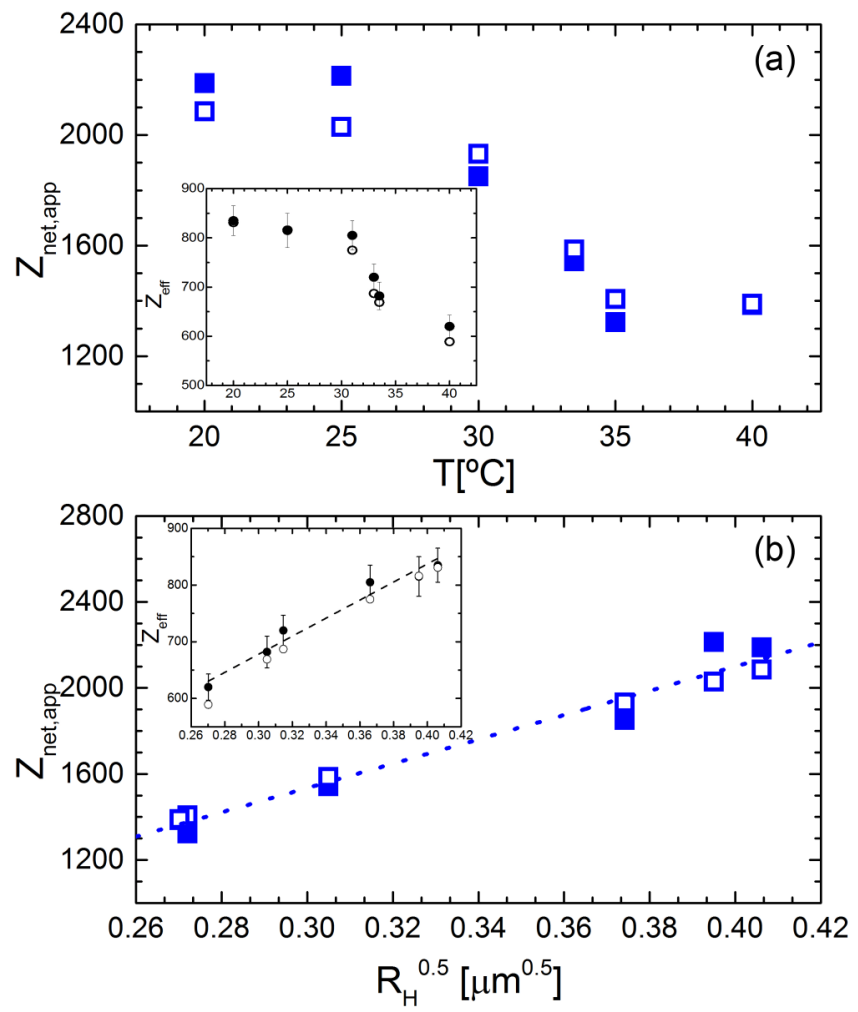

FIG. 5. (a) Comparison of experimental $Z_{\text {net, app }}$ obtained from the combination of conductivity and mobility experiments (solid squares) to $Z_{\text {net }}$ obtained in PB cell model calculations assuming a bare charge of 6000 (open squares). Inset: Comparison of experimental $Z_{\text {eff }}$ obtained from static light experiments (solid circles) to the results of the renormalized PB cell model calculations assuming a bare charge of 1150 (open circles). (b) Same data as in (a) reported as a function of the square root of the hydrodynamic radius. In both cases, main figure and inset, reporting the microgel charge as a function of $\sqrt{R_{h}}$ leads to a linearization of the data. The dashed and dotted lines are linear fits to the data.

is obtained by matching the linearized solution of Eq. (2) to the numerical solution at $R_{\text {cell }}$. The reader interested in the precise functional form of this linearized solution is referred to the original work by Alexander et al. [40]. Using this procedure the temperature dependence of the effective charges obtained in static light scattering is reasonably well captured, as shown in the inset of Fig. 5(a). However, in order to capture the experimental results quantitatively we need here to assume that the bare charge is $Z=1150$, significantly smaller than the bare charge assumed for the description of $Z_{\text {net,app. As mentioned }}$ previously, this large discrepancy is due to polymeric impurity; upon further purification $Z_{\text {eff }}$ is found to be of the order of $Z_{\text {net,app. }}$.

Finally, let us recall that the net charge is equal to the bare charge minus the number of counterions located within the microgel. The decrease of $Z_{\text {net,app }}$ and $Z_{\text {net }}$ with increasing temperature obtained in, respectively, experiment and theory thus indicates that the number of counterions trapped within the microgels increases as the microgels shrink, thereby canceling out the increase of electrostatic energy that the reduction in size involves. In fact, the decrease in $Z_{\text {net,app }}$ and $Z_{\text {net }}$ upon collapse of the microgel is best described by 
a square root dependence of $Z_{\text {net, app }}$ and, respectively, $Z_{\text {net }}$ on the hydrodynamic radius, as shown in Fig. 5(b). Interestingly, such square root dependence also applies for the decrease of $Z_{\text {eff }}$ with $R_{h}$, as shown in the inset of Fig. 5(b). Considering that the electrostatic energy of a spherical charge distribution is $W \sim Z^{2} / R$, this indicates that the electrostatic energy of the microgel is preserved when the size of the microgel is varied.

\section{CONCLUSIONS}

Our experimental investigations on the temperature dependence of electrostatic properties of PNiPAM microgels unambiguously show that the net charge of these microgels decreases upon collapse of the microgels. Assuming a given bare charge we find that the impact of the volume phase transition on the apparent net charge and the effective charge of the microgels is reasonably well reproduced by the PoissonBoltzmann cell model $[10,13]$. Within this model the microgel is considered a permeable sphere, in which the counterions are free to move even in the collapsed state of the microgels. That this assumption holds is justified by the fact that the collapsed state of PNiPAM still contains a significant amount of water [41].
Indeed, reducing significantly the volume available to the counterions within the microgel by shrinking the microgel dimension is predicted to lead to an increase of the electrostatic energy per particle [17]. By contrast we find that the electrostatic energy per particle remains constant upon changing the dimensions of our microgels, leading to a square root dependence of the net charge on the particle radius.

This remarkably simple finding should be useful to understand and control the phase behavior of other dilute, quasi-deionized microgel dispersions. To uncover the range of validity of this finding we plan to explore the size dependence of the microgel effective charge at larger microgel concentrations, in addition to investigating the effect of the bare charge and salt concentration on this dependence.

\section{ACKNOWLEDGMENTS}

We gratefully acknowledge financial support from the Swiss National Science Foundation (Grant No. 200020138212, M.B. and V.T.), from Conacyt-Mexico (Grants No. 166645 and No. 154733, C.H.P. and L.F.R.O.), from Ministerio de Economía y Competitividad, Plan Nacional de Investigación, Desarrollo e Innovación Tecnológica, Project No. FIS2016-80087-C2-2-P (M.Q.P.), and from the European Regional Development Fund (M.Q.P.).
[1] R. Pelton, Adv. Colloid Interface Sci. 85, 1 (2000).

[2] B. R. Saunders, N. Laajam, E. Daly, S. Teow, X. Hu, and R. Stepto, Adv. Colloid Interface Sci. 147-148, 251 (2009).

[3] J. Ramos, A. Imaz, J. Callejas-Fernandez, L. Barbosa-Barros, J. Estelrich, M. Quesada-Perez, and J. Forcada, Soft Matter 7, 5067 (2011).

[4] Y. Jiang, J. Chen, C. Deng, E. J. Suuronen, and Z. Y. Zhong, Biomaterials 35, 4969 (2014).

[5] W. Richtering and B. R. Saunders, Soft Matter 10, 3695 (2014).

[6] J. Estelrich, M. Quesada-Perez, J. Forcada, and J. CallejasFernandez, in Soft Nanoparticles for Biomedical Applications (The Royal Society of Chemistry, London, 2014), pp. 1-18.

[7] P. S. Mohanty and W. Richtering, J. Phys. Chem. B 112, 14692 (2008).

[8] A. R. Denton, Phys. Rev. E. 67, 011804 (2003).

[9] D. Gottwald, C. N. Likos, G. Kahl, and H. Löwen, J. Chem. Phys. 122, 074903 (2005).

[10] G. C. Claudio, K. Kremer, and C. Holm, J. Chem. Phys. 131, 094903 (2009).

[11] A. Moncho-Jordá, J. A. Anta, and J. Callejas-Fernández, J. Chem. Phys. 138, 134902 (2013).

[12] A. Moncho-Jordá, J. Chem. Phys. 139, 064906 (2013).

[13] M. Quesada-Perez and A. Martin-Molina, Soft Matter 9, 7086 (2013).

[14] S. Ahualli, A. Martín-Molina, and M. Quesada-Pérez, Phys. Chem. Chem. Phys. 16, 25483 (2014).

[15] M. M. Hedrick, J. K. Chung, and A. R. Denton, J. Chem. Phys. 142, 034904 (2015).

[16] P. González-Mozuelos, J. Chem. Phys. 144, 054902 (2016).

[17] I. Adroher-Benítez, S. Ahualli, A. Martín-Molina, M. QuesadaPérez, and A. Moncho-Jordá, Macromolecules 48, 4645 (2015).
[18] E. Daly and B. R. Saunders, Phys. Chem. Chem. Phys. 2, 3187 (2000).

[19] P. Holmqvist, P. S. Mohanty, G. Nägele, P. Schurtenberger, and M. Heinen, Phys. Rev. Lett. 109, 048302 (2012).

[20] H. Senff and W. Richtering, J. Chem. Phys. 111, 1705 (1999).

[21] G. Nägele, Phys. Rep. 272, 215 (1996).

[22] J. Liu, H. J. Schöpe, and T. Palberg, Part. Part. Syst. Charact. 17, 206 (2000).

[23] E. J. W. Verwey and J. Th. G. Overbeek, Theory of the Stability of Lyophobic Colloids (Elsevier, New York, 1948).

[24] K. Schätzel, J. Mod. Opt. 38, 1849 (1991).

[25] C. Urban and P. Schurtenberger, J. Colloid Interface Sci. 207, 150 (1998).

[26] H. J. Mos, C. Pathmamanoharan, J. K. G. Dhont, and C. G. de Kruif, J. Chem. Phys. 84, 45 (1986).

[27] L. B. Aberle, S. Wiegand, W. Staude, O. Methods, and U. Bremen, Colloid Polym. Sci. 104, 121 (1997).

[28] See correlator.com.

[29] S. H. Behrens and D. G. Grier, J. Chem. Phys. 115, 6716 (2001).

[30] H. J. Schope and T. Palberg, J. Phys.: Condens. Matter. 14, 45 (2002).

[31] D. Hessinger, M. Evers, and T. Palberg, Phys. Rev. E 61, 5493 (2000).

[32] S. Cukierman, Biophys. J. 78, 1825 (2000).

[33] R. H. Pelton, H. M. Pelton, A. Morphesis, and R. L. Rowell, Langmuir 5, 816 (1989).

[34] J. Zhou, J. Wei, T. Ngai, L. Wang, D. Zhu, and J. Shen, Macromolecules 45, 6158 (2012). 
[35] M. Stieger, W. Richtering, J. S. Pedersen, and P. Lindner, J. Chem. Phys. 120, 6197 (2004).

[36] M. Reufer, P. Díaz-Leyva, I. Lynch, and F. Scheffold, Eur. Phys. J. E 28, 165 (2009).

[37] M. Ledesma-Motolinía, M. Braibanti, L. F. Rojas-Ochoa, and Catalina Haro-Pérez, Colloids Surfaces, A 482, 724 (2015).
[38] J. P. Hansen and L. Verlet, Phys. Rev. 184, 151 (1969).

[39] H. Löwen, T. Palberg, and R. Simon, Phys. Rev. Lett. 70, 1557 (1993).

[40] S. Alexander, P. M. Chaikin, P. Grant, G. J. Morales, P. Pincus, and D. Hone, J. Chem. Phys. 80, 5776 (1984).

[41] C. Wu and X. Wang, Phys. Rev. Lett. 80, 4092 (1998). 